\title{
Diagnostic value of ultrasound score, color Doppler ultrasound RI and spiral CT for ovarian tumors
}

\author{
LI ZHOU, ZHIDONG XUAN and YUWANG
}

Department of Ultrasonography, Section 3, Cangzhou Central Hospital, Cangzhou, Hebei 061000, P.R. China

Received August 30, 2018; Accepted March 29, 2019

DOI: $10.3892 / 01.2019 .10215$

\begin{abstract}
Diagnostic value of ultrasound score, color Doppler ultrasound resistance index (RI) and spiral computed tomography (CT) for ovarian tumors was investigated. In total 224 patients pathologically diagnosed with ovarian tumor after operation in Cangzhou Central Hospital were selected, including 120 patients with benign ovarian tumor and 104 patients with malignant ovarian tumor. Patients with benign and malignant tumors were scored according to the ultrasound scoring criteria. The color Doppler ultrasound examination was performed and the corresponding RI was recorded. At the same time, 64-slice spiral CT was performed, and results were compared with the postoperative pathological diagnosis. Also, the sensitivity, specificity and accuracy of the combined application of the three methods were detected. The RI value of benign ovarian tumor was higher than that of malignant ovarian tumor, displaying statistically significant difference $(\mathrm{P}<0.05)$. The sensitivity, specificity and accuracy of the combined application of the three methods were 96.49 , 90.91 and $93.75 \%$, respectively. Ultrasound scoring, color Doppler ultrasound RI and 64-slice spiral CT have good diagnostic value for ovarian tumor, and the diagnostic accuracy rate of the combined application is higher. Therefore, different examination methods can be selected in clinic according to the different situations.
\end{abstract}

\section{Introduction}

Ovarian tumor is a common tumor in the female reproductive organ, and the incidence rate of malignant ovarian tumor ranks 3rd in gynecological tumors (1). The mortality rate for ovarian cancer is the highest in a variety of gynecological tumors, seriously threatening women's health and lives. The ovary is located deeply in the pelvic cavity, and there are often

Correspondence to: Dr Li Zhou, Department of Ultrasonography, Section 3, Cangzhou Central Hospital, 16 Xinhua Xi Road, Cangzhou, Hebei 061000, P.R. China

E-mail: gzz82q@163.com

Key words: ultrasound score, color Doppler ultrasound resistance index, 64-slice spiral computed tomography, ovarian tumor, diagnostic value no typical clinical manifestations when the tumor occurs, so most patients are in the late stage at the time of diagnosis. The 5-year survival rate of patients with advanced ovarian cancer is as low as $30-40 \%$, and the survival rate of early-stage patients is $\sim 90 \%(2,3)$. Therefore, the early diagnosis of ovarian cancer is the key to improving the survival rate of patients, which can provide a basis for treatment and prognosis.

Ultrasound, computed tomography (CT) and magnetic resonance imaging (MRI) are commonly-used imaging examination methods in clinic. Among them, MRI is difficult to be the preferred examination method and screening means for lesions, due to the high costs of equipment and examination. Ultrasound examination is currently the most commonly-used and convenient method in clinical screening of ovarian tumor, which can detect the morphology, size, capsule and wall thickness of ovarian tumors, the characteristics of echo in nodules and whether there are ascites, and can also preliminarily diagnose benign and malignant ovarian tumors according to the Finkler scoring system criteria (4). Color Doppler ultrasound flow imaging can also detect the blood flow resistance index (RI) and the velocity, based on the observation of the distribution characteristics and morphological features of tumor vessels, thus improving the diagnostic accuracy rate (5). RI can directly reflect the resistance against blood flow, and it is higher in benign ovarian tumors than in malignant ovarian tumors. Therefore, benign and malignant tumors can be differentially diagnosed by RI, but there is an overlap in the RI value between benign and malignant tumors (6). Moreover, the 64-slice spiral CT scan can position and qualitatively determine the tumor and indicate the lesion scope, because it can clearly display the position of the tumor and clarify the adjacent relationship between the tumor and pelvic organs (7-9).

In recent years, the diagnostic value of ultrasound combined with tumor markers in serum for ovarian tumors has been reported, but the detection of RI has not received much attention (10). Reports on the combination of several examination methods are rare, and no reports on CT diagnosis of ovarian tumors exist; specifically the application of 64-slice spiral CT in ovarian tumor has been less reported worldwide (11). Therefore, in this study, Finkler ultrasound score, color Doppler ultrasound RI and 64-slice spiral CT signs were compared with pathological diagnosis results, the sensitivity, specificity and accuracy were analyzed, and the diagnostic value of the combined application of the three methods for ovarian tumor was evaluated. 
Table I. Ultrasound scoring system.

Scoring criteria

Score

No echo in cysts with clear border, fibroma, and nodular cysts, such as hydrosalpinx 1

No echo in cysts with slightly irregular border, smooth cyst capsule with low echo 2

Low echo in cysts with slightly irregular border and no nodules (endometrioid tumor), 3 and postmenopausal non-echo cysts

Iso-echoic and non-specific ultrasound manifestations: solid ovarian enlargement, small cysts

with irregular border accompanied with internal echo reflex (hematoma or benign ovarian tumor)

Consistent manifestations with ovarian tumor: multiple isolated or irregular cystic masses

(7 points for a small number of nodules, and 8-9 for a large number of nodules)

Above characteristics accompanied with ascites

\section{Patients and methods}

Sample collection. A retrospective analysis was performed on 224 patients pathologically diagnosed with ovarian tumor after operation in Cangzhou Central Hospital (Cangzhou, China) from January 2010 to January 2014. There were 120 patients with benign tumor, aged $42 \pm 14.68$ years, and 104 patients with malignant tumor aged $53 \pm 12.73$ years. Inclusion criteria: patients were confirmed with ovarian tumor by postoperative pathology with complete clinical data and imaging. Exclusion criteria: patients with endometriosis, pelvic inflammation, adenomyosis, liver disease, ovarian hyperstimulation syndrome, uterine fibroids or diabetes mellitus were excluded. The study was approved by the Ethics Committee of Cangzhou Central Hospital (Cangzhou, China). Patients who participated in this research had complete clinical data. Signed informed consents were obtained from the patients or the guardians.

Instruments. Color Doppler ultrasonic apparatus was purchased from Siemens AG (Munich, Germany), and Philips Brilliance spiral CT 64-slice apparatus was purchased from Philips Medical Systems, Inc. (Bothell, WA, USA).

\section{Methods}

Color Doppler ultrasound examination. After filling of bladder, the probe scanned in multiple directions above the lower abdominal pubic symphysis of ovarian tumor patients under a supine position on the bed to observe the internal echo in tumor in the uterus and bilateral adnexa areas and whether it was accompanied by ascites, site of lesion, tumor morphology and boundary, whether there was isolation in the tumor and isolation thickness. Each tumor was scored based on the Finkler ultrasound scoring criteria (12), in which the score $<7$ points indicates a benign lesion and the score $\geq 7$ points indicates a malignant lesion. The ultrasound scoring system is shown in Table I.

$R I$ record. Color Doppler ultrasound examination was performed to observe the blood flow distribution around and in the tumor and the morphological characteristics of vessels, and to measure the blood flow RI around and in the tumor. $\mathrm{RI} \leq 0.45$ was taken as a diagnostic criterion for a malignant tumor, and RI $>0.45$ indicated a benign tumor. The blood flow
Table II. Clinicopathological data of patients (\%).

\begin{tabular}{lcc}
\hline Factor & $\mathrm{n}$ & Ratio \\
\hline Age (years) & & \\
$\geq 53$ & 118 & 52.68 \\
$<53$ & 106 & 47.32 \\
Clinical stage & & \\
T1+T2 & 68 & 65.38 \\
T3+T4 & 36 & 34.62 \\
Depth of infiltration & & \\
Muscular layer & 57 & 54.81 \\
Serosa & 47 & 45.19 \\
Degree of differentiation & & \\
High differentiation & 79 & 35.27 \\
Moderate-low differentiation & 145 & 64.73 \\
Lymph node metastasis & & \\
Yes & 40 & 38.46 \\
No & 64 & 61.54 \\
\hline
\end{tabular}

signal characteristics around and in the tumor can be divided into 3 types: type 1 , there is no blood flow signal around or in the tumor; type 2, there are punctiform and short line-like blood flow signals around the tumor or septum; and type 3, there are punctiform, strip, line or branched blood flow signals around the tumor and in the parenchymal area (13).

64-slice spiral CT examination. After fasting for $>12 \mathrm{~h}$ and filling of bladder until the need for micturition, scanning was performed for patients under the supine position from the inferior margin of pubis to the upper edge of mass. The whole abdominal scanning was performed for patients with large mass or abdominal metastasis. All patients received enhanced scanning, while some underwent delayed scanning, and the tumor was preliminarily positioned and qualitatively determined $(14,15)$. In the image analysis, benign and malignant ovarian tumors were identified, combined with AFP examination, by two imaging physicians with $>10$ years experience. Diagnostic criteria: positioning and qualitative judgment of ovarian tumors. CT diagnosis of ovarian cancer requires at least two doctors with the corresponding titles of radiology 
Table III. Ultrasound imaging of benign and malignant ovarian tumors [n (\%)].

\begin{tabular}{lccrr}
\hline Item & Benign $(\mathrm{n}=120)$ & Malignant $(\mathrm{n}=104)$ & $\chi^{2}$ & P-value \\
\hline Ultrasound characteristics & & & 29.680 & $<0.001$ \\
Cystic echo & $67(55.83)$ & $21(20.19)$ & 29.670 & $<0.001$ \\
Cystic-solid echo & $35(29.17)$ & $54(51.92)$ & 12.050 & $<0.001$ \\
Solid echo & $18(15.00)$ & $29(27.88)$ & 5.579 & 0.018 \\
Blood flow signal & $82(68.33)$ & $91(87.50)$ & 11.64 & $<0.001$ \\
\hline
\end{tabular}

Table IV. Diagnostic value of different imaging features in benign and malignant ovarian tumors.

\begin{tabular}{lccc}
\hline Feature & $\begin{array}{c}\text { Sensitivity } \\
(\%)\end{array}$ & $\begin{array}{c}\text { Specificity } \\
(\%)\end{array}$ & $\begin{array}{c}\text { Accuracy } \\
(\%)\end{array}$ \\
\hline Cystic echo & 55.83 & 79.81 & 66.96 \\
Cystic-solid echo & 29.17 & 48.08 & 37.95 \\
Blood flow signal & 68.33 & 12.50 & 42.41 \\
\hline
\end{tabular}

to evaluate the CT image, including the location, shape, size, cystic solidity, blood supply, degree of enhancement and peritoneal implantation (16).

Statistical analysis. Statistical Product and Service Solutions (SPSS) 16.0 software (Shanghai Cabit Information Technology Co., Ltd., Shanghai, China) was used for the statistical analysis of the results. Chi-square test was used for categorical data. The receiver operating characteristics (ROC) curve analysis was used for the diagnostic value of ultrasound score, color Doppler ultrasound RI, spiral CT, and the combined application of the three methods in ovarian tumors. ANOVA was used for the comparison between multiple groups and LSD test was the post hoc test. $\mathrm{P}<0.05$ was considered to indicate a statistically significant difference.

\section{Results}

General data. The clinicopathological data of 224 patients with ovarian cancer are shown in Table II.

Ultrasound manifestations of ovarian tumor in both groups. The cystic echo mostly appeared in benign ovarian tumors, while the cystic-solid echo mostly appeared in malignant ovarian tumors, displaying a statistically significant difference $(\mathrm{P}<0.05)$. The vascular resistance of benign ovarian tumors was larger than that of malignant tumors. It was easier to measure blood flow signals in malignant ovarian tumors. The difference between the two groups was statistically significant $(\mathrm{P}<0.001)$ (Table III). Compared with cystic echo and blood flow signals, the cystic echo, used to judge the benign and malignant ovarian cancer tumors, had the highest specificity and accuracy. Compared with cystic echo and cystic mixed echo, blood flow signal had the highest sensitivity in the assessment of the benign and malignant ovarian tumors (Table IV).
Table V. Diagnostic results of each examination method for ovarian tumors.

\begin{tabular}{lrcr}
\hline Pathological result & $\begin{array}{c}\text { Benign } \\
(\mathrm{n}=120)\end{array}$ & $\begin{array}{c}\text { Malignant } \\
(\mathrm{n}=104)\end{array}$ & Total \\
\hline Ultrasound score & & & \\
$\quad$ Benign & 102 & 12 & 114 \\
Malignant & 18 & 92 & 110 \\
RI & & & \\
Benign & 82 & 13 & 95 \\
Malignant & 38 & 91 & 129 \\
64-slice spiral CT & 106 & 13 & 119 \\
Benign & 14 & 91 & 105 \\
Malignant & & & \\
Combined application & 110 & 4 & 114 \\
Benign & 10 & 100 & 110 \\
Malignant & & & \\
\hline
\end{tabular}

RI, resistance index; CT, computed tomography.

Table VI. Comparisons of sensitivity, specificity and accuracy of each examination method for ovarian tumor (\%).

\begin{tabular}{lccc}
\hline Examination & Sensitivity & Specificity & Accuracy \\
\hline Ultrasound scoring & $89.47^{\mathrm{c}}$ & 83.64 & $86.61^{\mathrm{ac}}$ \\
RI & $86.32^{\mathrm{abd}}$ & $70.54^{\mathrm{a}}$ & $77.23^{\mathrm{abd}}$ \\
64-slice spiral CT & $89.08^{\mathrm{c}}$ & $86.67^{\mathrm{a}}$ & $87.95^{\mathrm{ac}}$ \\
Combined application & $96.49^{\mathrm{c}}$ & $90.91^{\mathrm{c}}$ & $93.75^{\mathrm{bcd}}$ \\
\hline
\end{tabular}

${ }^{\mathrm{a}} \mathrm{P}<0.05$, compared with combined application; ${ }^{\mathrm{b}} \mathrm{P}<0.05$, compared with 64-slice spiral $\mathrm{CT}$; ${ }^{\mathrm{c}} \mathrm{P}<0.05$, compared with $\mathrm{RI}$; ${ }^{\mathrm{d}} \mathrm{P}<0.05$, compared with ultrasound scoring. RI, resistance index; CT, computed tomography.

Diagnostic evaluation of different examination methods for ovarian tumors. The diagnostic results of the four methods in ovarian tumors were compared. With the pathological results as the gold standard, it was found that the sensitivity, specificity and accuracy of the combined application of ultrasound scoring, RI and 64-slice spiral CT in the diagnosis of benign ovarian tumor were higher than those of the single application. The diagnosis coincidence rate of RI for ovarian malignant 
Table VII. Comparison of the diagnosis coincidence rates of benign and malignant ovarian tumors for each examination method.

\begin{tabular}{lccrr}
\hline Method & Benign tumor $(\mathrm{n}=120)$ & Malignant tumor $(\mathrm{n}=104)$ & $\chi^{2}$ & P-value \\
\hline Ultrasound score & $102(85.00)$ & $92(88.46)$ & 0.576 & 0.448 \\
RI & $82(68.33)$ & $91(87.5)$ & 11.640 & 0.001 \\
64-slice spiral CT & $106(88.33)$ & $91(87.5)$ & 0.036 & 0.849 \\
Combined application & $110(91.67)$ & $100(96.15)$ & 1.915 & 0.167 \\
\hline
\end{tabular}

RI, resistance index; CT, computed tomography.

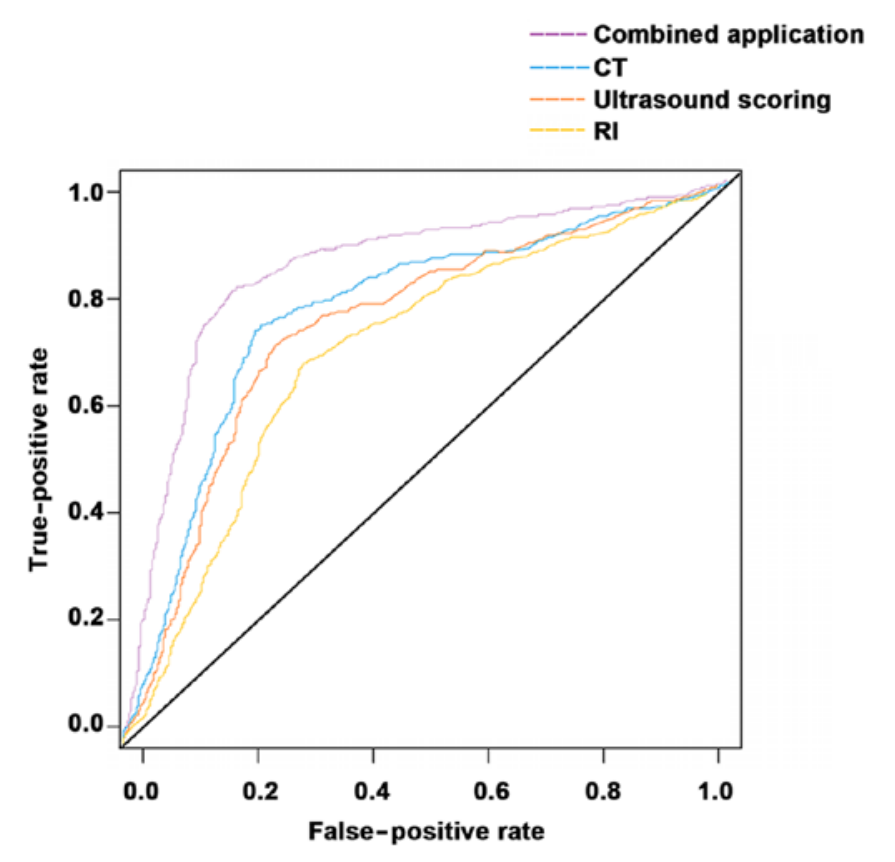

Figure 1. ROC curves of ultrasound scoring, RI, 64-slice spiral CT, and their combined application in the diagnosis of ovarian tumors. The area under the curve for the combined application is $>64$-slice spiral CT $>$ ultrasound scoring $>$ RI, with statistically significant differences $(\mathrm{P}<0.05)$. ROC, receiver operating characteristics; RI, resistance index; CT, computed tomography.

tumors was higher than that of benign tumors $(\mathrm{P}<0.05)$. There was no significant difference in the diagnosis coincidence rate of the other three methods for benign and malignant ovarian tumors $(\mathrm{P}>0.05)$ (Tables V-VII). The area under the ROC curve of the RI was smaller than that of the ultrasound score, which was smaller than that of the 64-slice spiral CT, and the area under the ROC curve of the 64-slice spiral CT was smaller than that of the combination of the three methods (Fig. 1).

\section{Discussion}

Patients with ovarian cancer mostly have no or mild symptoms in the early stage, and metastasis has often occurred with poor prognosis when treated. If patients can be diagnosed in the early stage, the therapeutic effect on ovarian tumor will be improved. Modern medical imaging techniques have provided examination means for the diagnosis of ovarian tumor, which are characterized by non-invasiveness and no pain and play important roles in the diagnosis of gynecological tumors (17). Although a variety of imaging methods have been widely used in the examination and diagnosis of ovarian tumor, the imaging application in ovarian tumor is diversified and variable due to the diverse sources of ovarian tumor cells and various tissue types. None of the imaging methods is able to diagnose accurately and qualitatively the ovarian mass in patients. In particular, the surrounding organs are squeezed and displaced when the ovarian tumor is $>50 \mathrm{~mm}$, so it is difficult to determine the source of the tumor and easy to confuse it with tumors from other sources (18).

In recent years, the measurement of RI has not attracted attention, and the combined application of ultrasound scoring, color Doppler ultrasound RI and spiral CT in ovarian tumors is less reported. CT diagnosis of ovarian tumors has been extensively reported worldwide, but not the application of 64-slice spiral CT (11). Therefore, in this study, the ultrasound score, RI and CT signs were compared with pathological diagnosis results, the differences in sensitivity and specificity were analyzed, and the value of each method in the differential diagnosis of ovarian tumor was evaluated, so as to provide insights for the selection and development of a clinical therapeutic regimen.

At present, ultrasound examination is the most common and convenient method in the clinical diagnosis of ovarian tumors. Observing the morphology and echo of an ovarian tumor and whether ascites are presented in ultrasound images can preliminarily determine the benign and malignant tumors according to the ultrasound scoring criteria. In this study, the ultrasound manifestation was mainly cystic echo in the benign tumor and cystic-solid echo in the malignant tumor. In some malignant tumors, the early morphology is regular and there is a lack of typical changes in the ultrasonogram, so it is difficult to accurately determine the benign and malignant ovarian tumors in the ultrasonogram of complex or early-stage malignant tumors only by using ultrasound examination. The sensitivity, specificity and accuracy of Finkler ultrasound scoring in the analysis of ovarian tumor were 89.47, 83.64 and $86.61 \%$, respectively. Alanbay et al (19) obtained research results that are basically consistent with ours. Fleischer and Brader (20) have shown that the pathological parameters of patients can be obtained through observing the characteristics of vascular distribution in tumor tissues via ultrasound examination. In the present study, blood flow signals could be detected around the benign tumor in 82 cases, in which the blood vessels were sparse with the type 1 characteristics of blood flow. Besides, blood flow signals could be detected in malignant ovarian tumors in 91 cases, in which there were abundant blood vessels and complex branches mainly distributed in and 
around the tumor with the type 3 characteristics of blood flow. The sensitivity, specificity and accuracy were 86.32, 70.54 and $77.23 \%$, respectively. The RI value of malignant ovarian tumor was lower than that of benign ovarian tumor, and the difference was statistically significant $(\mathrm{P}<0.001)$. According to the study of Kurjak et al (21), RI $\leq 0.4$ is the diagnostic criterion for malignant ovarian tumor. Waltmire et al (22) considered that $\mathrm{RI}<0.5$ can be taken as the criterion for positive prediction. The above conclusion is different from the results in this study, indicating that RI has no unified criteria for benign and malignant tumors, and the possible reason is that there are a variety of ovarian tumors with complex tissue components. Therefore, there is a partial overlap of RI value in benign and malignant ovarian tumors, and blood flow signals cannot be detected. In this study, 120 patients were pathologically diagnosed with benign ovarian tumor and 104 patients were diagnosed with malignant ovarian tumor. The sensitivity, specificity and accuracy of 64-slice spiral CT in the detection of ovarian tumor were $89.08,86.67$ and $87.95 \%$, respectively, which are consistent with the results of Taïeb et al (23) on the multi-slice CT in the diagnosis of ovarian tumor $(24,25)$. The sensitivity, specificity and accuracy of the combined application were 96.49, 90.91 and $93.75 \%$, respectively. The sensitivity, specificity and accuracy of the combined application of ultrasound scoring, RI and 64-slice spiral CT in the diagnosis of benign ovarian tumor were higher than those of the single application.

However, there were also some limitations in this study. Comparisons were not performed between 64-slice spiral CT and conventional CT, and between multi-planar reconstruction and curved planar reconstruction. Therefore, these issues will be further explored in a future study.

In conclusion, the cystic echo mostly appeared in benign ovarian tumor, while the cystic-solid echo mostly appeared in malignant ovarian tumor. The RI of benign ovarian tumor was higher than that of malignant ovarian tumor $(\mathrm{P}<0.001)$, so blood flow signals could be detected more easily in malignant ovarian tumor. The sensitivity and specificity in the diagnosis of ovarian tumor can be increased in the combined application of Finkler ultrasound scoring, RI value and 64-slice spiral CT scan, which can provide an effective basis for the early therapeutic regimen of ovarian tumor, thus improving the survival rate of patients.

\section{Acknowledgements}

Not applicable.

\section{Funding}

No funding was received.

\section{Availability of data and materials}

The datasets used and/or analyzed during the present study are available from the corresponding author on reasonable request.

\section{Authors' contributions}

LZ designed the study and drafted the manuscript. LZ and ZX collected and analyzed the general data. LZ and YW interpreted the results of the examinations. All the authors read and approved the final manuscript.

\section{Ethics approval and consent to participate}

The study was approved by the Ethics Committee of Cangzhou Central Hospital (Cangzhou, China). Patients who participated in this research had complete clinical data. Signed informed consents were obtained from the patients or the guardians.

\section{Patient consent for publication}

Not applicable.

\section{Competing interests}

The authors declare that they have no competing interests.

\section{References}

1. Curtin JP: Management of the adnexal mass. Gynecol Oncol 55: S42-S46, 1994.

2. Munkarah A, Chatterjee M and Tainsky MA: Update on ovarian cancer screening. Curr Opin Obstet Gynecol 19: 22-26, 2007.

3. Gansler T, Ganz PA, Grant M, Greene FL, Johnstone P, Mahoney M, Newman LA, Oh WK, Thomas CR Jr, Thun MJ, et al: Sixty years of CA. CA Cancer J Clin 60: 345-350, 2010.

4. Timmerman D, Testa AC, Bourne T, Ameye L, Jurkovic D, VanHolsbeke C, Paladini D, Van Calster B, Vergote I, Van Huffel S, et al: Simple ultrasound-based rules for the diagnosis of ovarian cancer. Ultrasound Obstet Gynecol 31: 681-690, 2008

5. Hale SA, Schonberg A, Badger GJ and Bernstein IM: Relationship between prepregnancy and early pregnancy uterine blood flow and resistance index. Reprod Sci 16: 1091-1096, 2009.

6. Hetland TE, Kærn J, Skrede M, Sandstad B, Tropé C, Davidson B and Flørenes VA: Predicting platinum resistance in primary advanced ovarian cancer patients with an in vitro resistance index. Cancer Chemother Pharmacol 69: 1307-1314, 2012.

7. Liang X, Jacobs R, Hassan B, Li L, Pauwels R, Corpas L, Souza PC, Martens W, Shahbazian M, Alonso A, et al: A comparative evaluation of cone beam computed tomography (CBCT) and multi-slice CT (MSCT) Part I. On subjective image quality. Eur J Radiol 75: 265-269, 2010.

8. Yang G, Bousse A, Toumoulin C and Shu H: A multiscale tracking algorithm for the coronary extraction in MSCT angiography. Conf Proc IEEE Eng Med Biol Soc 1: 3066-3069, 2006.

9. Becker N, Motsch E, Gross ML, Eigentopf A, Heussel CP, Dienemann H, Schnabel PA, Eichinger M, Optazaite DE, Puderbach M, et al: Randomized study on early detection of lung cancer with MSCT in Germany: Results of the first 3 years of follow-up after randomization. J Thorac Oncol 10: 890-896, 2015.

10. Deligeoroglou E, Eleftheriades M, Shiadoes V, Botsis D, Hasiakos D, Kontoravdis A and Creatsas G: Ovarian masses during adolescence: clinical, ultrasonographic and pathologic findings, serum tumor markers and endocrinological profile. Gynecol Endocrinol 19: 1-8, 2004.

11. Brodoefel H, Klumpp B, Reimann A, Fenchel M, Heuschmid M, Miller S, Schroeder S, Claussen C, Scheule AM and Kopp AF: Sixty-four-MSCT in the characterization of porcine acute and subacute myocardial infarction: Determination of transmurality in comparison to magnetic resonance imaging and histopathology. Eur J Radiol 62: 235-246, 2007.

12. Sankaranarayanan R and Ferlay J: Worldwide burden of gynaecological cancer: The size of the problem. Best Pract Res Clin Obstet Gynaecol 20: 207-225, 2006.

13. Kurjak A, Kupesic S, Anic T and Kosuta D: Three-dimensional ultrasound and power Doppler improve the diagnosis of ovarian lesions. Gynecol Oncol 76: 28-32, 2000.

14. Nolz R, Wibmer A, Beitzke D, Gentzsch S, Willfort-Ehringer A, Lammer J, Thurnher M and Schoder M: Carotid artery stenting and follow-up: Value of 64-MSCT angiography as complementary imaging method to color-coded duplex sonography. Eur J Radiol 81: 89-94, 2012. 
15. Brodoefel H, Klumpp B, Reimann A, Ohmer M, Fenchel M, Schroeder S, Miller S, Claussen C, Kopp AF and Scheule AM: Late myocardial enhancement assessed by 64-MSCT in reperfused porcine myocardial infarction: Diagnostic accuracy of low-dose CT protocols in comparison with magnetic resonance imaging. Eur Radiol 17: 475-483, 2007.

16. Khiewvan B, Torigian DA, Emamzadehfard S, Paydary K, Salavati A, Houshmand S, Werner TJ and Alavi A: An update on the role of PET/CT and PET/MRI in ovarian cancer. Eur J Nucl Med Mol Imaging 44: 1079-1091, 2017.

17. Ahamad A and Jhingran A: New radiation techniques in gynecological cancer. Int J Gynecol Cancer 14: 569-579, 2004.

18. McLaughlin JR, Risch HA, Lubinski J, Moller P, Ghadirian P, Lynch H, Karlan B, Fishman D, Rosen B, Neuhausen SL, et al; Hereditary Ovarian Cancer Clinical Study Group: Reproductive risk factors for ovarian cancer in carriers of BRCA1 or BRCA2 mutations: A case-control study. Lancet Oncol 8: 26-34, 2007.

19. Alanbay I, Akturk E, Coksuer H, Ercan M, Karasahin E, Dede M, Yenen MC, Ozan H and Baser I: Comparison of risk of malignancy index (RMI), CA125, CA 19-9, ultrasound score, and menopausal status in borderline ovarian tumor. Gynecol Endocrinol 28: 478-482, 2012.

20. Fleischer AC and Brader KR: Sonographic depiction of ovarian vascularity and flow: Current improvements and future applications. J Ultrasound Med 20: 241-250, 2001.
21. Kurjak A,Kupesic S,Sparac V,Prka Mand Bekavac I: The detection of stage I ovarian cancer by three-dimensional sonography and power Doppler. Gynecol Oncol 90: 258-264, 2003.

22. Waltmire CN, Alberts DS and Dorr RT: Sequence-dependent cytotoxicity of combination chemotherapy using paclitaxel, carboplatin and bleomycin in human lung and ovarian cancer. Anticancer Drugs 12: 595-602, 2001.

23. Taïeb S, Bonodeau F, Leblanc é, Vennin P, Fournier C and Besson P: Predictive value of preoperative abdominopelvic CT for optimal cytoreduction surgery in ovarian carcinoma. Bull Cancer 87: 265-272, 2000 (In French).

24. He B, Gong S, Hu C, Fan J, Qian J, Huang S, Cui L and Ji Y: Obscure gastrointestinal bleeding: Diagnostic performance of 64-section multiphase CT enterography and CT angiography compared with capsule endoscopy. Br J Radiol 87: 20140229, 2014.

25. Mathewson JW: Three dimensional imaging using 64 detector row multi-slice CT should be used more widely for the diagnosis and management of congenital heart disease. J Saudi Heart Assoc 22: 179-185, 2010.

This work is licensed under a Creative Commons Attribution-NonCommercial-NoDerivatives 4.0 International (CC BY-NC-ND 4.0) License. 